\title{
Toward a Cognitive Processing Theory of Player's Experience of Computer Mediated Environments
}

\section{Shulan Lu}

Derek Harter

Texas A \& M University-Commerce

Commerce, TX 75429, USA

Shulan.Lu@tamuc.edu

Derek.Harter@tamuc.edu
Permission to make digital or hard copies of part or all of this work for personal or classroom use is granted without fee provided that copies are not made or distributed for profit or commercial advantage and that copies bear this notice and the full citation on the first page. Copyrights for third-party components of this work must be honored. For all other uses, contact the Owner/Author.

Copyright is held by the owner/author(s).

CHI PLAY'16 Extended Abstracts, October 16-19, 2016, Austin, TX, USA ACM 978-1-4503-4458-6/16/10.

http://dx.doi.org/10.1145/2968120.2987742

\begin{abstract}
There is a large body of work in psychology demonstrating that aesthetic pleasure is a function of the perceiver's cognitive processing dynamics [6]. The current work aims to develop a theoretical account of the processing dynamics players experience in interacting with computer mediated environments. The present paper integrates the latest theories in Cognitive Science viewing the brain as a Bayesian hierarchical predictive machine, and develops insights into the presence illusion experienced in VR and other representational medium. Clark's [3] Bayesian hierarchical predictive processing theory indicates that reuse and redeployment of existing neuronal circuits would both be expected and would be a common and highly effective method to make (re)use of scarce cognitive resources. In the hierarchical predictive view of cognition, higher levels of the hierarchy have the goal of reducing prediction error on the generated error signals. We propose that users automatically adapt (typically lower) their expectations at the onset of interactions with virtual environments or other computer mediated environments. In the process of interaction, mismatches between expected and actual
\end{abstract}


signals produce prediction errors and lead to jagged processing fluency. Processing fluency is not presence, but presence entails processing fluency.

\section{Author Keywords}

presence; computer mediated environments; hierarchical predictive processing; redeployment and retooling; virtual environments, gameplay.

\section{ACM Classification Keywords}

H.1.2 User/Machine Systems; H.5.1 Multimedia Information Systems; I.2.6 Learning.

\section{A Brief Overview of Presence}

Research in computer mediated environments, including immersive virtual reality and desktop virtual environments, have demonstrated that people treat these environments as if they were real $[2,7,8,12]$

Many researchers in the virtual environments and telepresence community developed and tested various paradigms of VR devices and controllers. User experiences have two parts. Immersion refers to the system's technical capability in engaging the users' attention in the virtual interaction; while presence refers to the users' feeling of their bodies being transported into the virtual space [9]. A variety of measures have been used in determining the level of presence experienced: (a) subjective reports of users' conscious reflections of their experiences in the virtual environments; (b) behavioral measures of user responses to events, and (c) physiological patterns. Presence is perceived to be a multilevel representation, from unconscious arousal to conscious volitional responses.
The more consistent presence measures at different levels are, the greater the presence that will be experienced. For example, in the Milgram obedience study, where people agreed to deliver a lethal electric shock to strangers upon the request of an authority figure, was replicated in a virtual world. Some participants saw and heard the virtual learner's discomfort and pain responses, whereas others communicated with the virtual learner through a text interface only. Even knowing that the virtual learner cannot be truly harmed, people acted as if their actions had exerted effects on another real human being. Skin conductance level (i.e., overall sympathetic arousal) and skin conductance responses (i.e., transient arousal) were significantly different between the two conditions. Even though it was not necessary to wait between getting the learner's wrong answers and delivering the shock to the learner, participants in the learner visible condition waited significantly longer.

Other research has focused on training in virtual world simulations. The most familiar examples of these include simulations of real world tasks, such as flight simulators or surgical training [10]. Overall, the more presence trainees experience, the better the training effects and outcomes. The ultimate goal is to mesh virtual reality training to transfer learning outcomes to real world situations. However, some significant questions remain. Why can some minimal representations evoke realistic responses? Point lights of biological motion are one example, where the moving lights that mimic the motion of a human or animal are perceived to be real. This is in contrast with the development of high fidelity virtual environments. Consider another example, in the virtual reprisal of the Milgram study [11], even with a low fidelity visual 
appearance and behavior of a virtual character, participants experienced similar anxiety as they would with delivering a shock to a real human.

There is also a considerable amount of research done in investigating factors that could enhance gameplay experiences. For example, co-operative gameplay has been shown to enhance the flow that gets experienced during puzzle-based gameplay [4]. The realistic representation experienced via the use of Oculus Rift was shown to heighten the gameplay of first-person shooting game [13]. These are some examples of how video games have been used to explore aspects of presence in $\mathrm{HCI}$, but the results of these studies tend to conflict with each other and are subject to the characteristics of the particular study design, the characteristics of the gameplay, etc. At this stage, the community needs to look at the issue from the cognitive and information processing viewpoint and formulate questions in a coherent theoretical framework. This not only supports the systematic generation of research questions but also enables cross talk with other representational media in computer human interaction.

The goal of the computer mediated virtual environments is to enable the equivalence of the interactions in the real world. How do we define equivalence? The following sections attempt to integrate the latest theories in Cognitive Science, viewing the brain as a Bayesian hierarchical predictive machine, and develop insights into the presence illusion experienced in VR and other representational medium.

\section{Hierarchical Predictive View of Cognition, Prediction Error, and Presence}

The brain appears to be a type of hierarchically organized prediction machine [3]. In this probabilistic view of cognition, the top-down flow of probabilistic inference attempts to "explain away" any discrepancy or error it notices in the predictive models it makes of the lower-level signals. In states of great uncertainty, many such competing hypotheses can live and cooperate and compete, until evidence allows the burden of proof to shift in favor of one view or another.

An interesting conclusion of this hierarchical probabilistic view of cognition, is that the general processing mechanism being proposed means reuse and redeployment of circuits would both be expected and would be a common and highly effective method to make (re)use of scarce cognitive resources $[1,3,5]$.

Since higher-level layers are simply modeling and predicting an abstraction of the activity of a lower-level (and generating an error signal), it is most economical that other layers reuse an existing layer when dealing with a novel situation or in a new context.

The simplest and most obvious predictions to develop are those where motor actions should result in immediate effects. These tightly-coupled perception/action loops are important building blocks in our cognitive machinery. Failures at these levels have many more opportunities to disrupt higher-level conceptual models that are using them to maintain coherent predictions. That is to say, we would expect that when prediction errors occur at more fundamental levels of the hierarchy, they will possibly disrupt many higher-level theories (i.e., the best hypotheses of the real state of the world) the brain might be trying to 
maintain. When errors at these lower levels cannot be mitigated by the machinery, the result is a quick breakdown of being able to work within the virtual environment, and maintain feelings of presence with what is being experienced. There is evidence demonstrating that motor movements are a very powerful trigger (or disruptor) of feelings of presence in experiencing a virtual environment [14]. This indicates one component of presence, the user real time interaction experience, can be defined as perceptual prediction errors within a certain period of interactions.

The mind, however, is never a wholly unified entity. Many parts of the brain during such experiences are of course very aware of the (un)reality of the situation and the nature of the computer mediated virtual reality. This raises the question as to how users' cognitive machinery handles such inconsistency and its impact on user presence experience. We propose that users automatically adapt their expectations at the onset of interactions with virtual environments or other computer mediated environments. This reduces the prediction errors and enhances presence. This proposal could also explain why stimuli with low realism could trigger the experience of immersion.

Many other factors can influence how involved we get in a virtual experience. Our motivation, valence systems, involvement, personal history and personal differences can all factor into the presence experience. Top-down processes drive the cognitive experience to a coherent and meaningful interaction with the task or media we are consuming. When we are transported into the virtual environments, the top-down predictive models successfully suppress, ignore or cordon off inconsistent or contradictory information that can threaten to take us out of the feeling of presence. Conversely, the top-down processes can also work in the other direction, in order to enhance and detect such inconsistencies, thus allowing us to pick apart the experience and detect the unrealistic aspects of the computer mediated or fictional environment.

The better HCI designs and devices are at invoking users' personally, hedonically or motivationally marked experiential basis, and thus reducing prediction errors, the more likely we expect users will be to experience presence. Reduced prediction errors are crucial to user experiences of natural and productive human computer interaction. The perceptual prediction error as a computational measure of presence sheds light on the discrepancies many studies report between subjective reports of presence and real time behavioral measures of presence.

\section{Experimental Work In Progress}

Feelings of immersion and presence in virtual realities and virtual simulations are important for many reasons. In entertainment and computer games, immersion is generally seen as indicative of a good gaming experience, and more likely to keep players interested for longer periods, and keep them coming back to play the game and develop their skills within the game world.

Subjective reports are memory based conscious reflections of presence experience, whereas real time behavior measures are indicative of ongoing perceptual processing of interaction fluency. These two types of presence measures are interdependent with one another, however, they can be very different. For example, perceptual based presence could have little 
impact on memory based presence. Imagine a situation where users have small perceptual prediction errors except the last part of the interaction. This last chunk of interaction could trump previous feelings of presence when it comes to users' conscious reflections on presence. We could test this possibility by varying the properties of interactions that have greater impact on memory processes vs varying the properties of interactions that have greater impact on perceptual processes.

We have been investigating factors that support users in leveraging their existing real world representations when interacting with computer-mediated

environments. We have made significant progress in understanding how embodied a controller needs to be for users to invoke past experiential groundings. For example, in our experiments we demonstrated that users spent longer time in planning and executing vegetable cutting actions that are next to the fingertip when they used a knife like controller than merely gesturing knife cutting.

We also are making progress in developing and testing eye tracking measures of avatar embodiment. See Figure 1 as an example, where it shows the attentional allocation of a user in cutting celery in the virtual environment.

We have been developed real time temporal course measures of presence in terms of VR controller embodiment. We find that the first-fourth higher order derivatives of users' hand movements is indicative of the controller embodiment in the current research context.

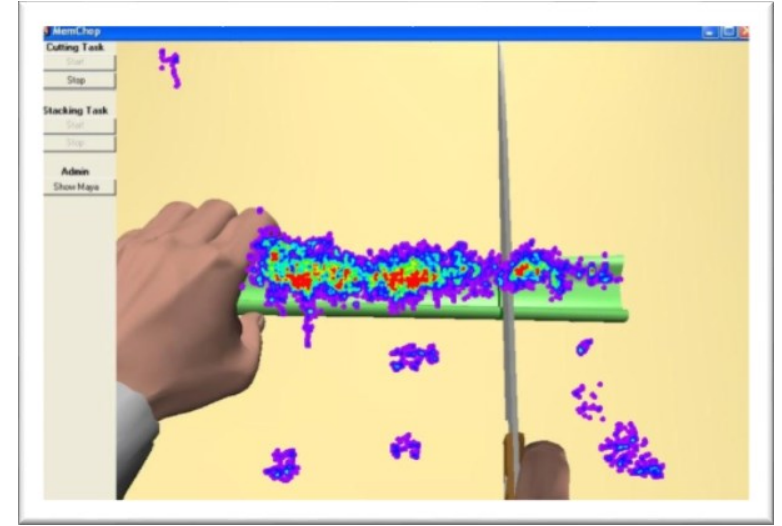

Figure 1: Attentional heat map in user gaming/training tasks with potentially risky virtual actions.

In short, this work aims to propose a computational model of presence, offers hypothesis to be tested in answering some of the thorny issues in virtual environments and computer mediated environments research. For example, we offered the theoretical insights into some of the critical ingredients to successful presence in computer mediated environments: a) realistic motion feedback loop; and b) the personalization of $\mathrm{HCI}$ designs and devices, because such personalization facilitates the alignment of users' mental states and thus reduces perceptual prediction errors.

\section{Acknowledgments}

This work is supported by a grant from US National Science Foundation (IIS-0742109, 0916749). Any opinions, findings, and conclusions or recommendations expressed in this paper are those of the authors and do not necessarily reflect the views of US National Science 
Foundation. Terry Rawlinson is our collaborator in developing the eye tracking measures of presence.

\section{References}

1. M.L. Anderson. 2010. Neural reuse: A fundamental organizational principle of the brain. Behavioral and Brain Sciences, 33: 245-266.

2. J. Blascovich, J. Bailenson. 2011. Infinite reality: Avatars, eternal life, new worlds, and the dawn of the virtual revolution. New York: HarperCollins.

3. A. Clark. 2013. Whatever next? Predictive brains, situated agents, and the future of cognitive science. Behavioral and Brain Sciences, 36, 3: 173.

4. C. Lee, P. Wyeth, D. Johnson, J. Hall. 2015. Flow during individual and cooperative gameplay. Proceedings of the 2015 Annual Symposium on Computer-Human Interaction in Play, 103-107.

5. S. Lu, D. Harter, P. Kosito, P. Kotturu. 2014. Developing low-cost virtual environments: How do effector and visual realism influence the perceptual grounding of actions? Journal of Cognitive Education and Psychology, 13: 3-18.

6. R. Reber, N. Schwarz, P. Winkielman. 2004. Processing Fluency and Aesthetic Pleasure: Is Beauty in the Perceiver's Processing Experience? Personality and Social Psychology Review, 8, 4: 364-382.

7. B. Reeves, C. Nass. 1996. Media equation: How people treat computers, television and new media like real people and places. CSLI Lecture Notes.
8. G. Riva, F. Mantovani. 2012. From the body to the tools and back: A general framework for presence in mediated interactions. Interacting with Computers, 24: 203-210.

9. M. V. Sanchez-Vives, M. Slater. 2005. From presence to consciousness through virtual reality. Nature Review Neuroscience, 6: 332-339.

10. N. Seymour. 2008. VR to OR: A review of the evidence that virtual reality simulation improves operating room performance. World Journal of Surgery, 32: 182-188.

11. M. Slater, et al. 2006. A virtual reprise of the Stanley Milgram obedience experiments. PLOS ONE, 1: 39. doi:10.1371/journal.pone.0000039.

12. M. Slater, M. V. Sanchez-Vives. 2014. Transcending the self in immersive virtual reality. Computer, July: $24-30$

13. C.T. Tan, T.W. Leong, S. Shen, C. Dubravs, C. Si. 2015. Exploring gameplay experiences on the Occulus Rift. Proceedings of the 2015 Annual Symposium on Computer-Human Interaction in Play, 253-263.

14. A. S. Won, J. D. Lee, J. N. Bailenson, J. Lanier. 2014. Homuncular flexibility in virtual reality. Journal of Computer-Mediated Communication. 